\title{
A New Finite Alphabet Based Blind Channel Estimation for OFDM Systems
}

\author{
Ibrahim Ghaleb \\ Alexandria University \\ Department of Electrical \\ Engineering \\ Alexandria, 21544 Egypt \\ Email: \\ ighaleb@netrabau.bau.edu.lb
}

\author{
Onsy Abdel Alim \\ Alexandria University \\ Department of Electrical \\ Engineering \\ Alexandria, 21544 Egypt \\ Email: onsy20@hotmail.com
}

\author{
Karim Seddik \\ Alexandria University \\ Department of Electrical \\ Engineering \\ Alexandria, 21544 Egypt \\ Email: karimgomaa@hotmail.com
}

\begin{abstract}
A new blind channel estimation algorithm is proposed based on the finite alphabet property of the transmitted symbols and the correlation between the channel attenuations at the adjacent subcarriers. The new method preserves the classical OFDM transmitter structure with Cyclic Prefix (CP) used in all standardized multicarrier systems (such as DAB, IEEE802.11a, Hiperlan/2, etc.) as well as the newly proposed OFDM with Zero Padding (ZP). The new method proves to provide an unbiased channel estimates and its performance is independent of the channel zero locations. The new method also works using only short data records for channels with short delay spread (e.g., indoor channels).
\end{abstract}

\section{INTRODUCTION}

There has recently been a large interest towards Orthogonal Frequency Division Multiplexing [1] for digital broadcasting (Terrestrial Audio Broadcasting (DAB), Video Broadcasting (DVB) ) and for mobile wireless broadband systems (Hiperlan/2, IEEE802.11a, and MMAC). The OFDM gained its popularity due to the ease with which it remedies the wireless multipath channel that causes inter-symbol interference (ISI) by converting the channel to a set of parallel flat fading channels across each subcarrier and hence simplifies the process of equalization at the receiver.

A way to avoid the need to know the channel is to use differential encoding (between the same subcarrier across blocks or between adjacent subcarriers in the same block) as proposed in the standards of DAB [1]. However, differential modulation results in 3 -dB loss compared to coherent detection. In order to reduce the spectral efficiency loss incurred by pilot symbols we can use blind or semi-blind methods for channel estimation which avoids (or at least reduces) the use of pilot symbols. A numerous blind channel estimation and blind equalization algorithms were developed in the literature (cf. [2] for a survey on these algorithms and the references therein).

In this paper, a new blind channel estimation algorithm is proposed based on the finite alphabet property of the transmitted symbols and the use of the same idea of differential encoding across subcarriers in the same block (the assumption that the channel attenuations at adjacent subcarriers are approximately the same). This new algorithm improves the performance compared to the subspace method of [3] for PSK modulation in the case of channels with short delay spread (e.g., indoor channels). In addition, the new algorithm is less complex specially for BPSK .

\section{Notations And System Model}

In the following, let $(\cdot)^{T}$ indicates the transpose, $(\cdot)^{\mathcal{H}}$ indicates the Hermitian transpose and (.) indicates frequency domain samples. The matlab's notation $\mathbf{A}(i: j, k: l)$ indicates the submatrix formed from the $i$ th to the $j$ th rows and the $k$ th to the $l$ th columns of the matrix $\mathbf{A}$ and $\left(\mathbf{V}^{\dagger}, \lambda_{\text {min }}\right)$ indicates the pseudo-inverse of matrix $\mathbf{V}$ neglecting the eigen values less than $\lambda_{\min } . \odot$ indicates element by element matrix multiplication. The system block diagram is shown in Figure (1). The data symbols are transmitted in blocks of size $M$ : $\tilde{\mathbf{s}}_{M}(i)=\left(\tilde{s}_{0}(i), \ldots, \tilde{s}_{M-1}(i)\right)^{T}$, where $i$ is the block index. These symbols are first precoded using the $M \times M$ IFFT matrix $\mathbf{F}_{M}^{\mathcal{H}}=$ $\mathbf{F}_{M}^{-1}$ with entry $(1 / \sqrt{M}) \exp (-j(2 \pi / M)(m-1)(n-1))$ at the $(m, n)$ element to yield the time domain block $\mathbf{s}_{M}(i)$. Then the cyclic prefix (CP) is appended between each block of symbols. The entries of the resulting redundant block $\mathbf{s}_{C P}(i)$ of length $P=M+L$ are transmitted sequentially through the frequency selective fading channel $\mathbf{h}$. The channel is modeled as an FIR filter with channel impulse response $\mathbf{h}=\left(h_{0}, \ldots \ldots, h_{L-1}\right)^{T}$ and define the $M \times 1$ vector $\mathbf{h}_{M}=\left(h_{0}, \ldots \ldots, h_{L-1}, 0, \ldots, 0\right)^{T}$. Let $\mathbf{I}_{C P}=\left(\mathbf{I}_{C}, \mathbf{I}_{M}\right)^{T}$ be the $P \times M$ matrix representing the cyclic prefix appending where $\mathbf{I}_{C}$ represents the last $L$ columns of $\mathbf{I}_{M}(M \times M$ identity matrix $)$, we also define the $P \times M$ matrix $\mathbf{F}_{C P}=\mathbf{I}_{C P} \mathbf{F}_{M}^{\mathcal{H}}$. Now the transmitted block is given by:

$$
\mathbf{s}_{C P}(i)=\mathbf{I}_{C P} \mathbf{F}_{M}^{\mathcal{H}} \tilde{\mathbf{s}}_{M}(i)=\mathbf{F}_{C P} \tilde{\mathbf{s}}_{M}(i) .
$$

At the receiver end, first the cyclic prefix is removed (and so is the IBI (inter block interference) assuming that the channel is shorter than the cyclic prefix). Denoting by $\mathbf{r}_{M}(i)$ the received $M \times 1$ vector after suppressing the CP: $\mathbf{r}_{M}(i)=$ $\left(r_{L}^{C P}(i), \ldots \ldots, r_{P-1}^{C P}(i)\right)^{T}$ and the noise is given by: $\mathbf{n}_{M}(i)=$ $\left(n_{L}(i), \ldots \ldots, n_{P-1}(i)\right)^{T}$. Due to the appending of the cyclic prefix the channel effect is circularized and it can be diagonalized using FFT at the receiver [4]. So we get:

$$
\begin{aligned}
\tilde{\mathbf{r}}_{M}(i) & =\mathbf{F}_{M} \mathbf{r}_{M}(i) \\
& =\sqrt{M} \operatorname{daig}\left(\mathbf{F}_{M} \mathbf{h}_{M}\right) \tilde{\mathbf{s}}_{M}(i)+\mathbf{F}_{M} \mathbf{n}_{M}(i) \\
& =\sqrt{M} \operatorname{daig}\left(\mathbf{F}_{M} \mathbf{h}_{M}\right) \tilde{\mathbf{s}}_{M}(i)+\tilde{\mathbf{n}}_{M}(i)
\end{aligned}
$$

And $\sqrt{M}$ daig $\left(\mathbf{F}_{M} \mathbf{h}_{M}\right)$ is a diagonal matrix with diagonal entries $(k, k)=\mathbf{H}\left(\exp \left(j \frac{2 \pi}{M} k\right)\right)$, where $\mathbf{H}\left(\exp \left(j \frac{2 \pi}{M} k\right)\right)$ is the channel attenuation at subcarrier $k$. We now define the channel attenuations at the subcarriers corresponding to the CIR $\mathbf{h}_{M}$ as: $\tilde{\mathbf{h}}_{M}=\sqrt{M}\left(\mathbf{F}_{M} \mathbf{h}_{M}\right)=\left(\mathbf{H}(0), \mathbf{H}\left(\exp \left(j \frac{2 \pi}{M}\right)\right), \ldots, \mathbf{H}\left(\exp \left(j \frac{2 \pi}{M}(M-\right.\right.\right.$ 1)) $))^{T}$, Equation (4) can now be reexpressed as:

$$
\tilde{\mathbf{r}}_{M}(i)=\operatorname{daig}\left(\tilde{\mathbf{h}}_{M}\right) \tilde{\mathbf{s}}_{M}(i)+\tilde{\mathbf{n}}_{M}(i)=\tilde{\mathbf{h}}_{M} \odot \tilde{\mathbf{s}}_{M}(i)+\tilde{\mathbf{n}}_{M}(i) .
$$




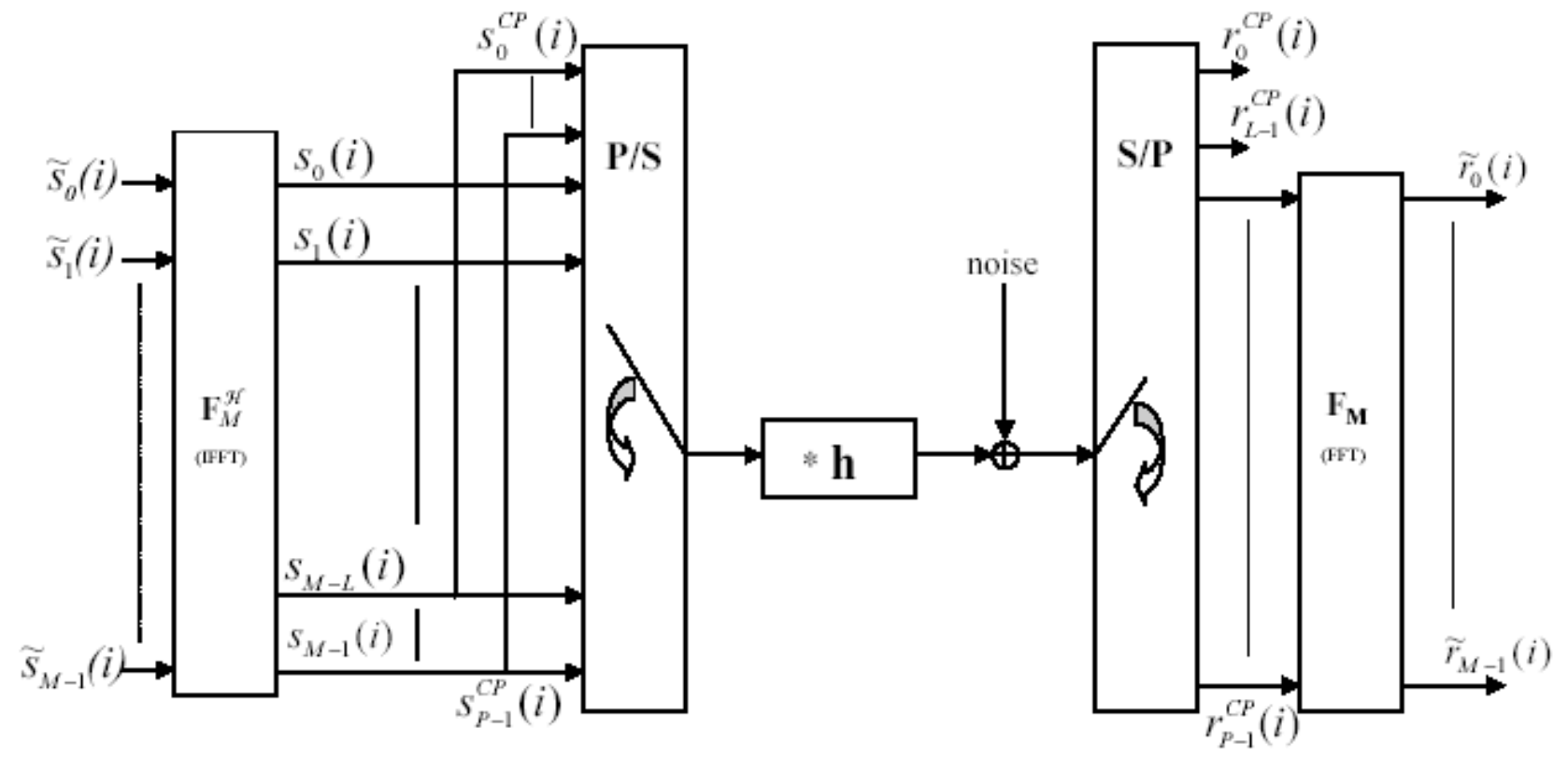

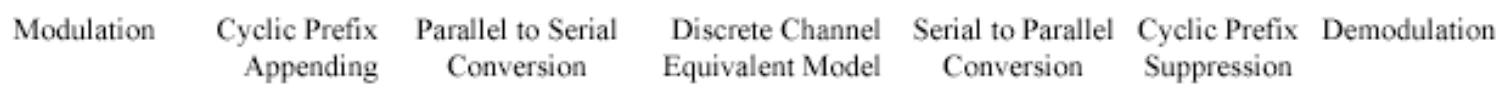

Figure 1: The OFDM-CP baseband system model

\section{Blind Channel Estimation}

In this section, a new blind channel estimation algorithm is proposed. The finite alphabet property of the transmitted data symbols is used to get estimates of $\mathbf{H}^{Q}\left(\exp \left(j \frac{2 \pi}{M} k\right)\right)$ and $k \in[0, M-1]$, where $Q$ is the constellation size. Based on the finite alphabet property of the transmitted symbols, a variety of algorithms were proposed in [5], [6]. All of these algorithms are very complex and cannot be used practically for channels of high order (for example in Hiperlan/2, the channel is assumed to be of length less than $L=16$ ). So we propose here a new algorithm that is computationally efficient and can be used in estimating channels of short delay spread. Then, under the assumption that the channel attenuations at adjacent subcarriers are approximately the same (used in differential encoding across subcarriers), one of the possible $\mathrm{Q}$ values of $\mathbf{H}\left(\exp \left(j \frac{2 \pi}{M} k\right)\right)$ is selected and the channel is estimated within a complex scale factor (inherent to all blind estimation algorithms).

The new algorithm is developed under the following assumptions (also used in [4]):

1. Symbols are drawn from finite alphabet set of size $Q$; i.e., $\tilde{s}_{k}(i) \in\left\{\xi_{q}\right\}_{q=1}^{Q}$.

2. Noise is zero-mean complex Gaussian noise and independent of the data.

For example, with BPSK and QPSK we have $Q=2$ and $Q=4$, respectively. For PSK modulation we have $\tilde{s}_{k}^{Q}(i)=1$ deterministically. Starting from $E\left\{\tilde{r}_{k}^{Q}(i)\right\}=\mathbf{H}^{Q}\left(\exp \left(j \frac{2 \pi}{M} k\right)\right) E\left\{\tilde{s}_{k}^{Q}(i)\right\}$ we deduce that (assuming noise free system):

$$
\mathbf{H}^{Q}\left(\exp \left(j \frac{2 \pi}{M} k\right)\right)=E\left\{\tilde{r}_{k}^{Q}(i)\right\}, \forall k \in[0, M-1] .
$$

The right hand side can be obtained for each $k$ deterministically for PSK modulation. The question is now how to get $\mathbf{H}\left(\exp \left(j \frac{2 \pi}{M} k\right)\right)$ from $\mathbf{H}^{Q}\left(\exp \left(j \frac{2 \pi}{M} k\right)\right)$. To express $\mathbf{H}^{Q}\left(\exp \left(j \frac{2 \pi}{M} k\right)\right)$ in terms of $\mathbf{h}$, we first define $\beta_{Q}^{T}=$ $\left(\beta_{0}, \ldots \ldots \ldots, \beta_{Q L}\right)^{M}=\mathbf{h}^{T} *_{Q} \mathbf{h}^{T}$ as the $Q$-fold convolution of the channel with itself. Since time domain convolution corresponds to multiplication in the frequency domain, we can write: $\quad \mathbf{H}^{Q}\left(\exp \left(j \frac{2 \pi}{M} k\right)\right)=\left.\mathbf{H}^{Q}(z)\right|_{z=\exp \left(j \frac{2 \pi}{M} k\right)}=\left(\beta_{0}+\right.$ $\left.\ldots \ldots \ldots \ldots+\beta_{Q L} z^{-Q L}\right)\left.\right|_{z=\exp \left(j \frac{2 \pi}{M} k\right)}$, and to determine the coefficients $\beta_{0}, \ldots \ldots \ldots \ldots, \beta_{Q L}$ uniquely from $\mathbf{H}^{Q}\left(\exp \left(j \frac{2 \pi}{M} k\right)\right)$, we need $Q L+1$ equations that can be available if $M \geq Q L+1$, thus enabling to find $\beta_{0}, \ldots \ldots \ldots \ldots, \beta_{Q L}$ uniquely. In practice (noisy case), $E\left\{\tilde{r}_{k}^{Q}(i)\right\}$ is replaced by statistical averaging and thus $\mathbf{H}^{Q}\left(\exp \left(j \frac{2 \pi}{M} k\right)\right)$ can be estimated as:

$$
\hat{\mathbf{H}}^{Q}\left(\exp \left(j \frac{2 \pi}{M} k\right)\right)=\frac{1}{N} \sum_{i=0}^{N-1} \tilde{r}_{k}^{Q}(i), \forall k \in[0, M-1]
$$

where $N$ is the total number of blocks used in the averaging. We next collect $\hat{\mathbf{H}}^{Q}\left(\exp \left(j \frac{2 \pi}{M} k\right)\right)$ from equation (7) in an $M \times 1$ vector: $\hat{\tilde{\mathbf{h}}}=\left(\hat{\mathbf{H}}^{Q}(0), \ldots \ldots, \hat{\mathbf{H}}^{Q}\left(\exp \left(j \frac{2 \pi}{M}(M-1)\right)\right)\right)^{T}$. Define the matrix $\mathbf{V}_{Q}$ to be a scaled version of the first $Q L+1$ columns of the FFT matrix $\mathbf{F}_{M}$ as $\mathbf{V}_{Q}=\sqrt{M} \mathbf{F}_{M}(:, 1: Q L+1)$ then we can estimate $\beta_{Q}$ by a simple matrix inversion as:

$$
\hat{\beta}_{Q}=\frac{1}{M} \mathbf{V}_{Q}^{\mathcal{H}} \hat{\tilde{\mathbf{h}}}
$$

\section{The New Algorithm}

We will start with $\hat{\mathbf{H}}^{Q}\left(\exp \left(j \frac{2 \pi}{M} k\right)\right)$ of equation $(7), \hat{\beta}_{Q}$ from equation (8) and proceed as follows to get the channel characterization in the frequency domain $\left(\hat{\mathbf{h}}_{M}\right)$ : 
1. Form the $M \times 1$ vector $\tilde{\mathbf{h}}_{P}$ with the $k$ th element given by $\tilde{\mathbf{h}}_{P}(k)=\left[\hat{\mathbf{H}}^{Q}\left(\exp \left(j \frac{2 \pi}{M}(k-1)\right)\right)\right]^{\frac{1}{Q}}$.

2. Form the $M \times Q$ matrix $\tilde{\mathbf{H}}_{P}=\left(\lambda_{0} * \tilde{\mathbf{h}}_{P}, \ldots, \lambda_{Q-1} * \tilde{\mathbf{h}}_{P}\right)$ that contains all the possible values of $\hat{\mathbf{H}}\left(\exp \left(j \frac{2 \pi}{M} k\right)\right)$ in the $k+1$ row, where $\lambda_{k}=\exp \left(j\left(\frac{2 \pi}{Q} k\right)\right)$.

3. Form the $M \times 1$ vector $\tilde{\mathbf{h}}_{\text {freq }}$ as:

(a) $\tilde{\mathbf{h}}_{\text {freq }}(1)=\tilde{\mathbf{H}}_{P}(1,1)$

(b) For $x=2: M$,

$$
\tilde{\mathbf{h}}_{\text {freq }}(x)=\operatorname{argmin}_{n}\left|\tilde{\mathbf{h}}_{\text {freq }}(x-1)-\tilde{\mathbf{H}}_{P}(x, n)\right|^{2}
$$

i.e., selecting the closest value of the possible estimates of $\hat{\mathbf{H}}\left(\exp \left(j \frac{2 \pi}{M} m\right)\right)$ to the previously selected $\hat{\mathbf{H}}\left(\exp \left(j \frac{2 \pi}{M}(m-\right.\right.$ 1))) (the assumption that the channel attenuations at adjacent subcarriers are approximately the same).

4. Place a sliding window of size $L+1$ (since we need only $L+1$ samples in the frequency domain to estimate the channel in the time domain) and estimate the channel as:

(a) $\mathbf{h}_{x}=\left(\mathbf{V}_{x}^{\dagger}, 0.01\right) \tilde{\mathbf{h}}_{\text {freq }}(x: x+L, 1), x=1, \ldots, M-L$ where $\mathbf{V}_{x}=\mathbf{F}_{M}(x: x+L+1,1: L)$.

(b) estimate the channel as:

$$
\hat{\mathbf{h}}=\operatorname{argmin}_{\mathbf{h}_{x}}\left|\hat{\beta}_{Q}^{T}-\mathbf{h}_{x}^{T} *_{Q} \mathbf{h}_{x}^{T}\right|^{2}
$$

and define the $(L+1) \times 1$ vector $\tilde{\mathbf{h}}_{r}=\tilde{\mathbf{h}}_{\text {freq }}\left(x_{\text {best }}: x_{\text {best }}+\right.$ $L$ ), where $x_{\text {best }}$ is $x$ that results in the minimum norm from equation (9).

5. $\tilde{\mathbf{h}}_{r}$ now contains $L+1$ elements that characterizes the channel at $L+1$ frequencies and to complete the estimation process we search over the remaining $M-L-1$ frequencies. At each remaining frequency, each of the possible $Q$ values of $\hat{\mathbf{H}}\left(\exp \left(j \frac{2 \pi}{M} k\right)\right)$ is tried and the same condition in equation (9) is used. Thus $(M-L-1)$ iterations are required to complete the estimation process.

In step 4 , we neglected the eigen values less than 0.01 due to the bad condition of the matrix to be inverted $\left(\mathbf{V}_{x}\right)$.

\section{Simulation Results}

In the first example we will use an OFDM system with $M=32$ and $L=8$ (CP length). We will use the measured channel $\mathbf{h}=(0.66,-0.46,-0.28,-0.22,0.12)^{T}$ of [5], [8]. With BPSK signaling and similar to [5] and [8], we overestimated the channel order as $L=8$. Because for QPSK signaling we need $M \geq Q L+1$, we adopted $L=7$ to assure a fair comparison. Figure (2) shows the performance of our method compared to the subspace method of [3], [8] for both BPSK and QPSK modulation using $N=300$ OFDM symbols. We use the Timedomain Normalized Mean Square Error (TD NMSE) defined as:

$$
\text { TD NMSE }=\frac{\sum_{k=0}^{L}\left|h_{k}-\hat{h}_{k}\right|^{2}}{\sum_{k=0}^{L}\left|h_{k}\right|^{2}} \text { as a performance measure. }
$$

The improvement in performance is clear in Figure (2) for both the BPSK and QPSK modulation schemes.

In the second example we will use the Hiperlan $/ 2$ system parameters $(M=64$ carriers and $L=16$ (CP length)). We will use BPSK modulation. Figures (3) and (4) depict the performance of the proposed method compared to the subspace method of [3], [8] for BPSK over exponential power delay profile channels with delay spread $=1$ sample (typical delay spread for channel model A in Hiperlan/2) and delay spread $=3$ sample (typical delay spread for channel model $\mathrm{C}$ in Hiperlan/2) using 500 randomly generated channels assuming exponential power delay profile and static channel. The improvement in the performance is clear in both figures. Figure (3) shows that the proposed method can give the same performance of the subspace method using only a small number of received blocks and this is clear in Figure (5) which shows the convergence of both methods for channels with delay spread $=1$ sample at $\mathrm{SNR}=30 \mathrm{~dB}$. This is an attractive property which indicates that this new algorithm can be applied for channels that change rapidly.

\section{Conclusion}

A new channel estimation algorithm is proposed, based on finite alphabet property of the transmitted symbols and the same idea used in differential encoding across subcarriers in the same block (the assumption that the channel attenuations at adjacent subcarriers are approximately the same). The new method is proved to improve the performance compared to the subspace methods for PSK modulation in the case of channels with short delay spread (e.g., indoor channels).

The subspace method achieves a performance that is constellation independent but the new method is less complex specially for BPSK and gives a performance that is independent of the channel zero locations which cause failure of the subspace method of [3] if one (or more) channel zero is located on a subcarrier. The new method can also be used with the newly proposed OFDM with Zero Padding (ZP) [7]. Also the subspace requires at least $(P+M)$ received blocks to converge while the new algorithm can estimate the channel from only one block in the noise free case and converges faster for the noisy case as clear in Figure (5).

\section{REFERENCES}

[1] Z. Wang and G. B. Giannakis, "Wireless multicarrier communications: Where Fourier meets Shannon," IEEE Signal Process. Mag., vol. 17, No. 3, pp. 29-48, May 2000.

[2] H. Liu, G. Xu, L. Tong, and T. Kailath, "Recent developments in blind channel equalization : From cyclostationnarity to subspaces," Signal Processing, vol. 50, pp. 83-99, April 1996.

[3] B. Muquet, M. de Courville and P. Duhamel, "Subspace-based blind and semi-blind channel Estimation for OFDM systems," IEEE Trans. on Signal Processing, vol. 50, pp. 1699-1712, July 2002.

[4] G. H. Golub and C. F. Van Loan, "Matrix Computations," John Hopkins University Press, 1996.

[5] S. Zhou, G. B. Giannakis, and A. Scaglione, "Long codes for generalized FH-OFDMA through unknown multipath channels," IEEE Trans. Commun., vol. 49, pp. 721-733, Apr. 2001.

[6] S. Zhou and G. B. Giannakis, "Finite-alphabet based channel estimation for OFDM and related multicarrier systems," IEEE Trans. Commun., vol. 49, pp. 1402-1414, Aug. 2001.

[7] B. Muquet, M. de Courville, P. Duhamel, and G. B. Giannakis, "OFDM with Trailing Zeros Versus OFDM with Cyclic Prefix: Links, Comparisons and Application to the HIPERLAN/2 System," in Proc. Int. Conf. on Communications, New Orleans, LA,pp. 1049-1053, June 2000.

[8] B. Muquet, M. de Courville, P. Duhamel, and V. Buenac, "A subspace based blind and semi-blind channel identification method for OFDM systems," in Proc. IEEE-SP Workshop on Signal Proc. Advances in Wireless Comm., Annapolis, MD,pp. 170-173, May 9-12, 1999. 


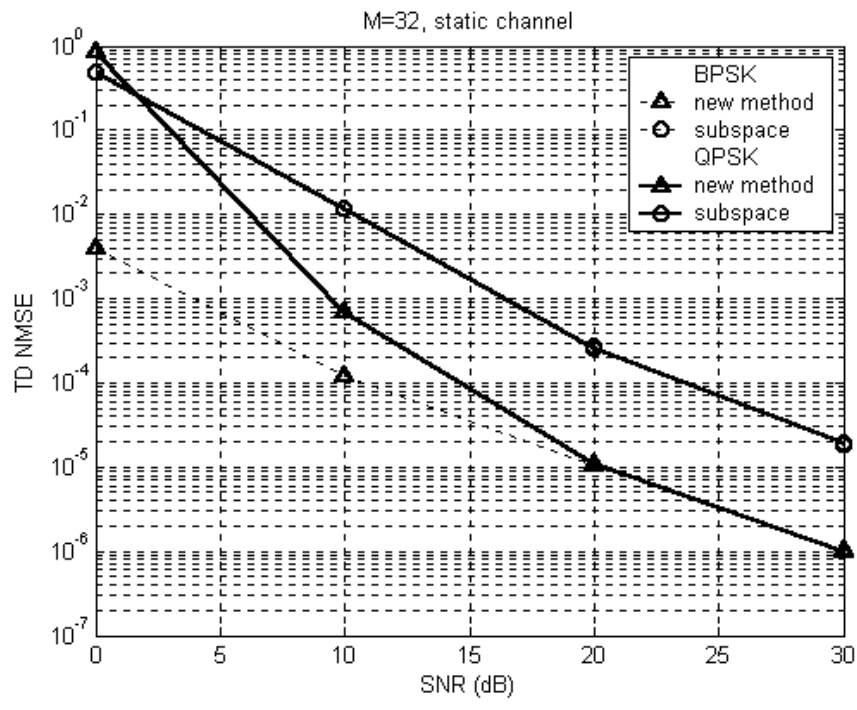

Figure 2: Performance of the new algorithm compared to the subspace method.

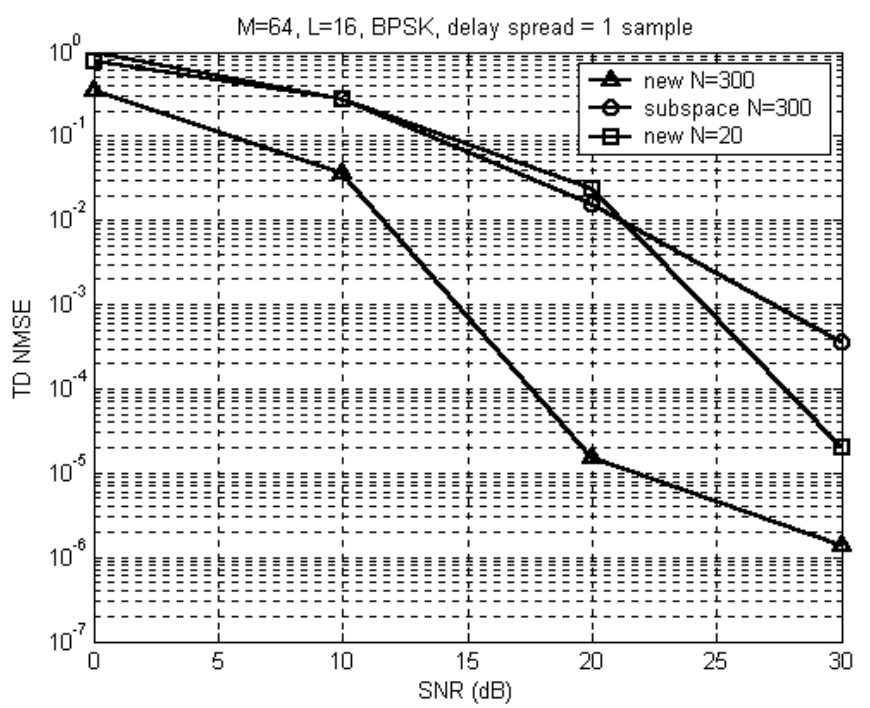

Figure 3: Channel TD NMSE for delay spread = 1 sample and BPSK modulation.

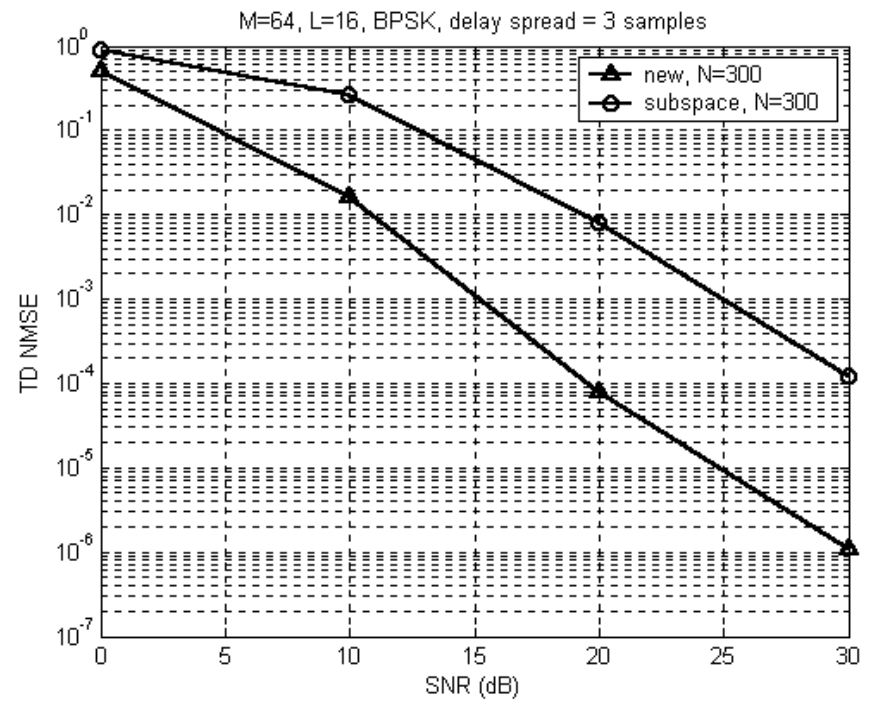

Figure 4: Channel TD NMSE for delay spread $=3$ sample and BPSK modulation.

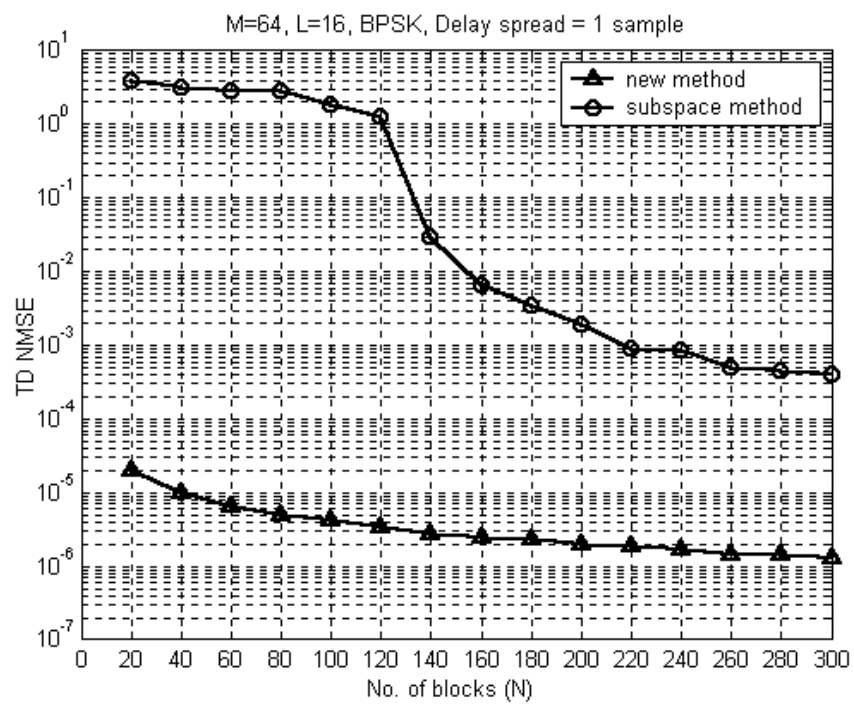

Figure 5: Convergence of the new algorithm compared to the subspace method. 